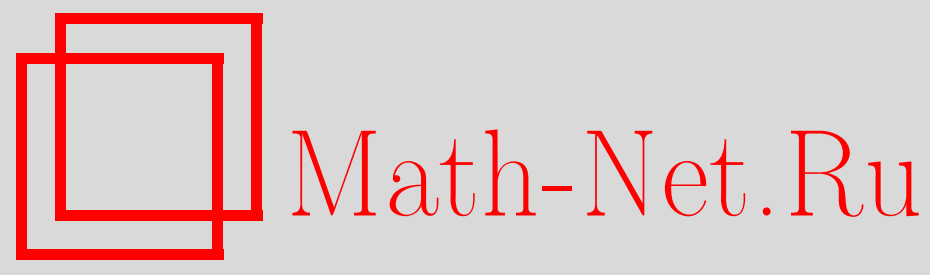

И. Д. Тверитинов, Несколько замечаний о представлении бесконечномерной симплектической группы и о построении метаплектической группы, Матем. заметки, 2004, том 75, выпуск 6, 861-876

DOI: https://doi.org/10.4213/mzm81

Использование Общероссийского математического портала Math-Net.Ru подразумевает, что вы прочитали и согласны с пользовательским соглашением http://www.mathnet.ru/rus/agreement

Параметры загрузки:

IP : 44.207 .124 .84

26 апреля 2023 г., 12:59:08 


\title{
НЕСКОЛЬКО ЗАМЕЧАНИЙ О ПРЕДСТАВЛЕНИИ БЕСКОНЕЧНОМЕРНОЙ СИМПЛЕКТИЧЕСКОЙ ГРУППЫ И О ПОСТРОЕНИИ МЕТАПЛЕКТИЧЕСКОЙ ГРУППЫ
}

\author{
И. Д. Тверитинов
}

\begin{abstract}
Построено явное проективное представление подгруппы симплектической группы в пространстве Винера-Сигала-Фока, а также расширение Макки подгрупп симплектической группы.

Библиограффия: 9 названий.
\end{abstract}

Введение. В работе рассматриваются конструкции, связанные с построением проективного представления симплектической групш бесконечномерного симплектического пространства и унитарным представлением расширений симплектической групшы и ее подгрупп. Для построения проективного представления используется техника преобразований Боголюбова. Поскольку в бесконечномерном пространстве, в отличие от конечномерного, существуют неэквивалентные невырожденные гауссовские меры, оказывается необходимым рассматривать не всю симплектическую групп, а некоторые ее подгрушы, для всех элементов которых существуют унитарные преобразования Боголюбова. В первой части работы найдены явные формулы для операторов проективного представления одной из таких подгрупп симплектической групш, причем операторы этого проективного представления, действуюшие в проективном пространстве, построенном по пространству Винера-Сигала-Фока, порождаются унитарными преобразованиями пространства Винера-Сигала-Фока (унитарными преобразованиями Боголюбова). Показано также, что в конечномерном случае используемая ниже конструкция представления (по-видимому, она впервые появилась в работе [1]) приводит к представлению, не эквивалентному представлению, рассмотренному в книге [2].

Далее в работе рассматриваются расширения Макки подгрупп симплектической групп; точнее, для каждого элемента этой подгруппы указывается определенное унитарное преобразование Боголюбова, порождающее соответствуюший оператор проективного представления (действуюший в проективном пространстве, построенном по пространству Винера-Сигала-Фока) и по коциклу, возникшему в результате выбора унитарных преобразований Боголюбова, строится расширение Макки. Используемая

Работа выполнена при поддержке Российского фонда фундаментальных исследований, грант № 02-01-01074. 
операторнозначная функция, определенная на подгруппе симплектической группы (элемент подгрупшы переходит в преобразование Боголюбова), продолжается до унитарного представления расширения Макки, построенного по коциклу, соответствующему этой операторнозначной функции. Доказано отсутствие подгрупп в расширении Макки, имеющих двузначную проекцию. В то жевремя в работе приводится класс подгрупा симплектической группы (в конечномерном случае сама симплектическая групша принадлежит этому классу), для которых существует двулистное накрытие с указанньп выше свойством (подгруппа расширения Макки имеет двузначную проекцию). Поэтому из результатов работы вытекают результаты, аналогичныеполученным в книге [2], в которой построено двулистное накрытие конечномерной симплектической группы, так назьваемая метаплектическая групша. Все результаты этой работы получены путем предъявления явных конструкций.

1. Предварительные сведения. Пусть $Q$ и $P$ - две копии действительного гильбертова пространства, $H$ - комплексное гильбертово пространство, полученное путем наделения пространства $Q \times P$ комплексной структурой, задаваемой оператором

$$
J:(q, p) \in Q \times P \mapsto(-p, q) \in Q \times P
$$

$B(\cdot, \cdot)$ - билинейная антисимметричная форма на $H \times H$, определяемая как мнимая часть эрмитова скалярного произведения в $H$. Группа обратимых преобразований (операторов) в $H$, сохраняющих форму $B(\cdot, \cdot)$, называется симплектической әруппой (см. [3], [4]). Симплектические преобразования удобно представлять матрицами

$$
\left(\begin{array}{ll}
\alpha & \beta \\
\gamma & \delta
\end{array}\right),
$$

где $\alpha, \beta, \gamma, \delta$ - операторы, действуюшие соответственно из $Q$ в $Q$, из $P$ в $Q$, из $Q$ в $P$, из $P$ в $P$. В дальнейшем для обозначения такой матрицы будем использовать символ $(\alpha, \beta, \gamma, \delta)$. Из равенства $B\left(\left(p_{1}, q_{1}\right),\left(p_{2}, q_{2}\right)\right)=\left\langle p_{1}, q_{2}\right\rangle-\left\langle p_{2}, q_{1}\right\rangle$, где $\langle\cdot, \cdot\rangle-$ скалярное произведение в $Q$, получается следующее условие сохранения формы $B(\cdot, \cdot)$ при преобразовании $(\alpha, \beta, \gamma, \delta)$ :

$$
\delta^{*} \alpha-\beta^{*} \gamma=\mathrm{id}, \quad \delta^{*} \beta=\beta^{*} \delta, \quad \gamma^{*} \alpha=\alpha^{*} \gamma .
$$

Обозначим через $L_{2}\left(Q, \mu_{1 / 2}\right)$ пространство, полученное в результате пополнения пространства цилиндрических функций, определенных на $Q$, с квадратом, интегрируемьп по гауссовой цилиндрической мере с корреляционным оператором $I / 2$ (гильбертово пространство $L_{2}\left(Q, \mu_{1 / 2}\right)$ назьвают пространством Винера-Сигала-Фока). Эта гауссова цилиндрическая мера не является $\sigma$-аддитивной на алгебре цилиндрических подмножеств пространства $Q$. Стоит отметить, что эта мера будет $\sigma$-аддитивной, если считать множества из ее области определения подмножествами пространства $Q_{-}$, полученного пополнением $Q$ по норме $\langle T(\cdot), T(\cdot)\rangle^{1 / 2}$, где $T$ - инъективньй оператор Гильберта-Шмидта в пространстве $Q$ (подробнее см. [5]). Для всякого $a \in Q$ определим неограниченные операторы $\widehat{q}_{a}, \widehat{p}_{a}$ в пространстве $L_{2}\left(Q, \mu_{1 / 2}\right)$ следующим образом:

$$
\widehat{q}_{a}: f \mapsto[x \mapsto\langle x, a\rangle f(x)], \quad \widehat{p}_{a}: f \mapsto[x \mapsto-\operatorname{id} f(x)(a)+i\langle x, a\rangle f(x)]
$$


Эти операторы образуют представление Шрёдингера алгебры Гейзенберга. Симплектическая группа естественным образом действует на операторы представления Шрёдингера:

$$
\left(\begin{array}{c}
\widehat{q}_{a} \\
\widehat{p}_{a}
\end{array}\right) \mapsto\left(\begin{array}{c}
\widehat{q}_{\alpha^{*}(a)}+\widehat{p}_{\beta^{*}(a)} \\
\widehat{q}_{\gamma^{*}(a)}+\widehat{p}_{\delta^{*}(a)}
\end{array}\right)
$$

ОПРЕДЕЛЕНИЕ 1. Унитарный оператор $V_{A}$ такой, что для всех $a \in Q$ на общей области существенной самосопряженности операторов представления Шрёдингера вьполнено равенство

$$
\left(\begin{array}{c}
V_{A}^{-1} \circ \widehat{q}_{a} \circ V_{A} \\
V_{A}^{-1} \circ \widehat{p}_{a} \circ V_{A}
\end{array}\right)=\left(\begin{array}{l}
\widehat{q}_{\alpha^{*}(a)}+\widehat{p}_{\beta^{*}(a)} \\
\widehat{q}_{\gamma^{*}(a)}+\widehat{p}_{\delta^{*}(a)}
\end{array}\right),
$$

назьвается унитарным преобразованием Боголюбова, соответствующим симплектическому преобразованию.

Только что определенное преобразование операторов представления Шрёдингера также называют преобразованиями Боголюбова. Так как оператор $V_{A}$, удовлетворяющий тем же соотношениям, что и унитарные преобразования Боголюбова, определяется (если он существует) с точностью до комплексного скалярного множителя, не равного нулю, которьй при условии существования унитарных преобразований Боголюбова может быть выбран так, что $V_{A}$ станет унитарньм (см. [4]), то, рассматривая подгруппу симплектической групп, для всех элементов которой существуют унитарные преобразования Боголюбова, можно определить ее проективное представление. Так как каждый оператор этого проективного представления порождается унитарным оператором в пространстве Винера-Сигала-Фока (соответствующим преобразованием Боголюбова), то можно говорить о многозначном отображении (с точностью до комплексного множителя, равного по модулю единице) в группу унитарных операторов пространства Винера-Сигала-Фока, которое естественно назвать унитарным проективным представлением.

2. Явный вид некоторых преобразований Боголюбова. Симплектическому преобразованию $A=(\mathrm{id}, 0, b, \mathrm{id})$, где $b$ - самосопряженньй оператор ГильбертаШмидта, соответствует унитарное преобразование Боголюбова

$$
V_{A}: f \mapsto\left[x \mapsto \exp \frac{i\langle b(x), x\rangle}{2} f(x)\right] .
$$

Из того, что $b$ - оператор Гильберта-Шмидта, следует возможность продолжения функции $\langle b(x), x\rangle$ на $Q$ - до измеримой (см. [5]). Если $A=(0, \mathrm{id},-\mathrm{id}, 0)$, то $V_{A}$ - преобразование Фурье-Винера (см. [5]), определяемое на цилиндрических функциях из пространства Шварца формулой

$$
V_{A}: f \mapsto\left[x \mapsto \exp \frac{\langle x, x\rangle}{2} \int_{P} e^{-i\langle x, y\rangle} f(y) d \mu_{1}(y)\right] .
$$

Если $A=\left(d^{-1}, 0,0, d^{*}\right)$, где оператор $d$ околоединичньй (это означает, что $d-I-$ ядерньй оператор), то

$$
V_{A}: f \mapsto\left[x \mapsto \sqrt{|\operatorname{det}(d)|} \exp \left(\frac{\langle x, x\rangle}{2}-\frac{\langle d(x), d(x)\rangle}{2}\right) f(d(x))\right] .
$$


Если $d-I$ является оператором Гильберта-Шмидта, то для оператора $V_{A}$ можно аналогичным образом указать явную формулу.

Пусть $A=(\alpha, \beta, \gamma, \delta)$, где оператор $\beta$ обратимьй и околоединичньй. Тогда $A$ можно представить в виде произведения симплектических преобразований рассмотренного выше типа. Действительно, преобразуя равенство

$$
\left(d^{-1}, 0,0, d^{*}\right) \cdot(\mathrm{id}, 0, c, \mathrm{id}) \cdot(0, \mathrm{id},-\mathrm{id}, 0) \cdot(\mathrm{id}, 0, b, \mathrm{id})=(\alpha, \beta, \gamma, \delta),
$$

получаем

$$
\left(d^{-1} b, d^{-1}, d^{*} c b-d^{*}, d^{*} c\right)=(\alpha, \beta, \gamma, \delta)
$$

что эквивалентно

$$
d=\beta^{-1}, \quad b=\beta^{-1} \alpha, \quad c=\beta^{*} \delta .
$$

Для того чтобы при таком выборе параметров $a, b, c$ определяемые ими операторы оказались симплектическими, требуется, чтобы были выполнены равенства $c^{*}=c, b^{*}=b$. Справедливость первого равенства следует из того, что $A$ - симплектическое преобразование. Пользуясь (2) и учитывая, что симплектические преобразования образуют группу и что все множители в (1) кроме, быть может, сомножителя, содержащего параметр $b=\beta^{-1} \cdot \alpha$, являются симплектическими преобразованиями, получаем второе равенство. Таким образом, доказана следующая

Теорема 1. Пусть $A=(\alpha, \beta, \gamma, \delta)$ - симплектическое преобразование, причем $\alpha, \delta$ - операторы Гильберта-Шмидта и $\beta$ - околоединичный обратимый оператор. Тогда для иилиндрических функиий из класса Швариа унитарное преобразование Боголюбова $V_{A}$ задается формулой

$$
\begin{aligned}
& V_{A}: f \mapsto\left[x \mapsto \frac { \operatorname { e x p } ( \langle x , x \rangle / 2 ) } { \sqrt { | \operatorname { d e t } ( \beta ) | } } \int _ { P } \operatorname { e x p } \left(i \left\{\frac{\left\langle\delta \beta^{-1} x, x\right\rangle}{2}\right.\right.\right. \\
&\left.\left.\left.-\left\langle\beta^{-1} x, y\right\rangle+\frac{\left\langle\beta^{-1} \alpha y, y\right\rangle}{2}\right\}\right) f(y) d \mu_{1}(y)\right] .
\end{aligned}
$$

Покажем, что от требования обратимости оператора $\beta$ можно отказаться (по-прежнему предполагая, что $\beta$ - околоединичньй оператор, а $\alpha, \delta$ - операторы ГильбертаШмидта). Апшроксимируем $A$ симплектическими операторами

$$
A_{n}=\left(\alpha+\frac{a \gamma}{n} \beta+\frac{a \delta}{n}, \gamma, \delta\right)
$$

с невырожденньг $\beta$ ( $a$-ортогональный проектор на $\left.\operatorname{Ker} \beta^{*}\right)$. Из равенств

$$
\begin{gathered}
\delta^{*}\left(\alpha+\frac{a \gamma}{n}\right)-\left(\beta^{*}+\frac{\delta^{*} a}{n}\right) \gamma=\delta^{*} \alpha-\beta^{*} \gamma=\mathrm{id} \\
\delta^{*}\left(\beta+\frac{a \delta}{n}\right)=\beta^{*}+\frac{\delta^{*} a \delta}{n}=\left(\beta+\frac{a \delta}{n}\right)^{*}, \\
\gamma^{*}\left(\alpha+\frac{a \gamma}{n}\right)=\alpha^{*} \gamma+\frac{\gamma^{*} a \gamma}{n}=\left(\alpha+\frac{a \gamma}{n}\right)^{*} \gamma
\end{gathered}
$$


следует, что преобразование $A_{n}$ симплектическое. Так как для околоединичных операторов обратимость эквивалентна отсутствию ядра, то получаем, что оператор $\beta+a \delta / n$ обратим. Применяя теорему 1 , для цилиндрической функции из пространства Шварца получаем следующее:

$$
\begin{aligned}
V_{A}(f)(x)= & \lim _{n \rightarrow \infty} V_{A_{n}}(f)(x) \\
= & \lim _{n \rightarrow \infty} \frac{1}{\sqrt{|\operatorname{det}(\beta+a \delta / n)|}} \exp \frac{\langle x, x\rangle}{2} \int_{P} \exp \left(i \left\{\frac{\left\langle\delta(\beta+a \delta / n)^{-1} x, x\right\rangle}{2}\right.\right. \\
& \left.\left.-\left\langle\left(\beta+\frac{a \delta}{n}\right)^{-1} x, y\right\rangle+\frac{\left\langle(\beta+a \delta / n)^{-1}(\alpha+a \gamma / n) y, y\right\rangle}{n}\right\}\right) f(y) d \mu_{1}(y) .
\end{aligned}
$$

Сделав замену $y=\left(\beta^{*}+\delta^{*} a\right) z$ и учитывая, что

$$
\frac{d \mu^{t^{-1}}}{d \mu}(x)=|\operatorname{det}(t)| \exp \frac{\langle x, x\rangle-\langle t x, t x\rangle}{2}
$$

где оператор $(t-I)$ ядерньй, а оператор $t$ обратимый, получаем

$$
\begin{aligned}
V_{A}(f)(x)= & \lim _{n \rightarrow \infty} \sqrt{|\operatorname{det}(\beta+a \delta)|} \sqrt{n}^{\operatorname{dim}\left(\operatorname{Ker} \beta^{*}\right)} \exp \frac{\langle x, x\rangle}{2} \\
= & \int_{\operatorname{Im} \beta} \int_{\operatorname{Ker} \beta^{*}} \exp \left(i \left\{\frac{\left\langle a x+p x,\left(\beta^{*}+\delta^{*} a / n\right)^{-1} \delta^{*} x\right\rangle}{2}\right.\right. \\
& \left.\left.\quad-\left\langle p x+a x, z_{1}+n z_{2}\right\rangle+\frac{\left\langle(\alpha+\gamma / n)\left(\beta^{*} z_{1}+\delta^{*} z_{2}\right), z_{1}+n z_{2}\right\rangle}{2}\right\}\right) \\
& \quad \times \exp \frac{\langle z, z\rangle-\left\langle\left(\beta^{*}+\delta^{*} a\right) z,\left(\beta^{*}+\delta^{*} a\right) z\right\rangle}{2} f\left(\beta^{*} z_{1}+\delta^{*} z_{2}\right) d \mu_{1}\left(z_{2}\right) d \mu_{1}\left(z_{1}\right),
\end{aligned}
$$

где $p$ - ортогональньй проектор на $\operatorname{Im} \beta$, a $z_{1}=p(z), z_{2}=a(z)$.

ЛЕмма 1. Справедливы равенства

$$
\begin{gathered}
\left\langle a x+p x,\left(\beta^{*}+\frac{\delta^{*} a}{n}\right)^{-1} \delta^{*} x\right\rangle=n\langle a x, a x\rangle+\langle p x, p \delta u\rangle, \\
\left\langle\alpha \beta^{*} z_{1}+\alpha \delta^{*} z_{2}, z_{1}+n z_{2}\right\rangle=\left\langle\alpha \beta^{*} z_{1}, z_{1}\right\rangle+n\left\langle z_{2}, z_{2}\right\rangle+\left\langle\alpha \delta^{*} z_{2}, z_{1}\right\rangle .
\end{gathered}
$$

Здесь вектор и определяется равенством $p x=\beta u$. 
ДокаЗАТЕЛЬСтво. Так как

$$
\begin{aligned}
\left\langle a x+p x,\left(\beta^{*}+\frac{\delta^{*} a}{n}\right)^{-1} \delta^{*} x\right\rangle & =\left\langle a x+p x,\left(\beta^{*}+\frac{\delta^{*} a}{n}\right)^{-1} \delta^{*}(a x+p x)\right\rangle \\
& =\langle a x+p x, n a x\rangle+\left\langle a x+p x,\left(\beta^{*}+\frac{\delta^{*} a}{n}\right)^{-1} \delta^{*} p x\right\rangle \\
& =n\langle a x, a x\rangle+\left\langle a x+p x,\left(\beta^{*}+\frac{\delta^{*} a}{n}\right)^{-1} \delta^{*} \beta u\right\rangle \\
& =\langle n a x, n a x\rangle+\left\langle a x+p x,\left(\beta^{*}+\frac{\delta^{*} a}{n}\right)^{-1} \beta^{*} \delta u\right\rangle \\
& =n\langle a x, a x\rangle+\left\langle a x+p x,\left(\beta^{*}+\frac{\delta^{*} a}{n}\right)^{-1} \beta^{*}(a \delta u+p \delta u)\right\rangle \\
& =n\langle a x, a x\rangle+\langle a x+p x, p \delta u\rangle=n\langle a x, a x\rangle+\langle p x, p \delta u\rangle
\end{aligned}
$$

и $A^{-1}=\left(\delta^{*},-\beta^{*},-\gamma^{*}, \alpha^{*}\right)$, то, используя условия симплектичности, получаем, что для всех $x \in \operatorname{Ker} \beta^{*}$ вьполнено $\delta \alpha^{*} x=x$. Таким образом,

$$
\begin{aligned}
\left\langle\alpha \beta^{*} z_{1}+\alpha \delta^{*} z_{2}, z_{1}+n z_{2}\right\rangle & =\left\langle\alpha \beta^{*} z_{1}, z_{1}\right\rangle+n\left\langle\alpha \beta^{*} z_{1}, z_{2}\right\rangle+n\left\langle\alpha \delta^{*} z_{2}, z_{2}+\frac{1}{n} z_{1}\right\rangle \\
& =\left\langle\alpha \beta^{*} z_{1}, z_{1}\right\rangle+n\left\langle z_{2}, z_{2}\right\rangle+\left\langle\alpha \delta^{*} z_{2}, z_{1}\right\rangle
\end{aligned}
$$

Лемма доказана.

Используя лемму 1 , получаем

$$
\begin{aligned}
V_{A}(f)(x)= & \lim _{n \rightarrow \infty} V_{A_{n}}(f)(x) \\
= & \lim _{n \rightarrow \infty} \exp \frac{\langle x, x\rangle}{2} \sqrt{|\operatorname{det}(\beta+a \delta)|} \sqrt{n} \operatorname{dim} \operatorname{Ker} \beta \\
& \times \int_{\operatorname{Im} \beta} \int_{\operatorname{Ker} \beta^{*}} \exp \frac{i n\left\langle z_{2}-a x, z_{2}-a x\right\rangle}{2} \\
& \times \exp \left(i \left\{\frac{\langle p x, p \delta u\rangle}{2}-\left\langle p x, z_{1}\right\rangle+\frac{\left\langle\alpha \beta^{*} z_{1}, z_{1}\right\rangle}{2}+\frac{\left\langle\alpha \delta^{*} z_{2}, z_{1}\right\rangle}{2}\right.\right. \\
& \left.\left.+\frac{\left\langle a \gamma\left(\beta^{*} z_{1}+\delta^{*} z_{2}\right), z_{2}\right\rangle}{2}\right\}\right) \\
& \times \exp \frac{\left\langle z_{1}+z_{2}, z_{1}+z_{2}\right\rangle-\left\langle\left(\beta^{*}+\delta^{*} a\right) z,\left(\beta^{*}+\delta^{*} a\right) z\right\rangle}{2} \\
& \times f\left(\beta^{*} z_{1}+\delta^{*} z_{2}\right) d \mu_{1}\left(z_{2}\right) d \mu_{1}\left(z_{1}\right) .
\end{aligned}
$$

Лемма 2. Пусть $f(\cdot)$ - функиия из пространства Швариа. Тогда

$$
\lim _{n \rightarrow \infty}\left(\frac{\sqrt{n}}{\sqrt{2 \pi}}\right)^{k} \int_{\mathbb{R}^{k}} \exp \frac{i\langle x, x\rangle n}{2} f(x) d x=\exp \frac{i \pi k}{4} f(0) .
$$

Применяя лемму 2 , получаем следующую теорему. 
Теорема 2. Если $A=(\alpha, \beta, \gamma, \delta)$ - симплектическое преобразование, причем оператор $\beta$ околоединичный, а $\alpha, \delta$ - операторы Гильберта-Шмидта, то для иилиндрической функиии $f(\cdot)$ из класса Швариа унитарное преобразование Боголюбова задается следующей формулой:

$$
\begin{aligned}
& V_{A}(f)(x)=\exp \frac{i \pi \operatorname{dim} \operatorname{Ker} \beta}{4} \sqrt{|\operatorname{det}(\beta+a \delta)|} \exp \frac{\langle p x, p x\rangle}{2} \\
& \quad \times \int_{\operatorname{Im} \beta} \exp \left(i\left\{\frac{\langle p x, p \delta u\rangle}{2}-\langle p x, z\rangle+\frac{\left\langle z, \alpha \beta^{*} z+\alpha \delta^{*} a x\right\rangle}{2}+\frac{\left\langle\gamma \beta^{*} z+\gamma \delta^{*} a x, a x\right\rangle}{2}\right\}\right) \\
& \quad \times \exp \frac{\langle z, z\rangle-\left\langle\left(\beta^{*}+\delta^{*} a\right)(z+a x),\left(\beta^{*}+\delta^{*} a\right)(z+a x)\right\rangle}{2} f\left(\beta^{*} z+\delta^{*} a x\right) d \mu_{1}(z) .
\end{aligned}
$$

Для случая конечномерного симплектического пространства аналогичная теорема доказана в [6].

3. Унитарные преобразования Боголюбова, порожденные унитарными симплектическими преобразованиями. Симплектическое преобразование вещественного пространства $H$ является унитарным преобразованием комплексного пространства $H$ тогда и только тогда, ког да оно $C$-линейно или, что то же самое, когда $\alpha=\delta$, $\beta=-\gamma$. Ранее рассмотренный класс симплектических преобразований содержит не все унитарные преобразования, но известен явньй вид для унитарных преобразований Боголюбова в пространстве Фока, отвечающих унитарньм преобразованиям симплектического пространства. Пространство $F(H)=\bigoplus_{n=0}^{\infty}\left(\bigotimes^{n} H\right)$ с нормой

$$
\|\cdot\|_{F(H)}=\sum_{n=0}^{\infty}\|\cdot\|_{H^{n}}
$$

называется пространством Фока (подробнее см. [7], [8]). Для каждого $f \in H$ положим $a(f): F(H) \rightarrow F(H)$ так, чтобы

$$
a^{*}(f):\left(a, z_{1}^{1}, \ldots, z_{1}^{n} \vee \cdots \vee z_{n}^{n}, \ldots\right) \mapsto\left(0, a f, f \vee z_{1}^{1}, \ldots, f \vee z_{1}^{n} \vee \cdots \vee z_{n}^{n}, \ldots\right),
$$

где

$$
z_{1} \vee \cdots \vee z_{n}=\frac{1}{\sqrt{n !}} \sum_{\sigma \in S_{n}} z_{\sigma(1)} \otimes \cdots \otimes z_{\sigma(n)}
$$

Для операторов $a(f), a^{*}(f)$ верны канонические коммутационные соотношения (KKC)

$$
[a(f), a(g)]=\left[a^{*}(f), a^{*}(g)\right]=0, \quad\left[a(f), a^{*}(g)\right]=\langle f, g\rangle_{C} I,
$$

где $[A, B]=A B-B A$. Для операторов

$$
a_{1}(x \times y)=\frac{\widehat{q}_{x}-i \widehat{q}_{y}+\widehat{p}_{y}+i \widehat{p}_{x}}{\sqrt{2}}, \quad a_{1}^{*}(x \times y)=\frac{\widehat{q}_{x}+i \widehat{q}_{y}+\widehat{p}_{y}-i \widehat{p}_{x}}{\sqrt{2}},
$$


действующих в пространстве Винера-Сигала-Фока и порождающих алгебру, порожденную операторами $\widehat{q}_{a}, \widehat{p}_{a}$, выполняются KKС. Унитарноепреобразование $U$ пространства $H$ порождает преобразование операторов

$$
\left(\begin{array}{c}
a(f) \\
a^{*}(f)
\end{array}\right) \mapsto\left(\begin{array}{c}
a(U(f)) \\
a^{*}(U(f))
\end{array}\right)
$$

Соответствующее преобразование Боголюбова

$$
V_{U}:\left(a, z_{1}^{1}, \ldots, z_{1}^{n} \vee \cdots \vee z_{n}^{n}, \ldots\right) \mapsto\left(a, U\left(z_{1}^{1}\right), \ldots, U\left(z_{1}^{n}\right) \vee \cdots \vee U\left(z_{n}^{n}\right), \ldots\right)
$$

Отсюда следует, что если

$$
U=\exp i \phi_{1} P_{1}+\cdots+\exp i \phi_{n} P_{n}
$$

где $P_{i}$ - ортогональные проекторы на попарно ортогональные подпространства, то $V_{U}$ обладает полной системой собственных функций, которая может быть описана следующим образом.

Если $\left\{e_{n}^{i}\right\}$ - ортонормированный базис в $P_{i}(H)$, то $\left\{e_{n}^{i}\right\}$ - полная система собственных функций для $U$. Легко видеть, что

$$
\left(0,0, \ldots, e_{k_{1,1}}^{1} \vee \cdots \vee e_{k_{1, m_{1}}}^{1} \vee e_{k_{2,1}}^{2} \vee \cdots \vee e_{k_{2, m_{2}}}^{2} \vee \cdots \vee e_{k_{n, m_{n}}}^{n}, 0\right)
$$

- собственные функции оператора $V_{U}$ с собственными значениями $\exp i\left(m_{1} \phi_{1}+\cdots\right.$ $\left.+m_{n} \phi_{n}\right)$. Добавляя к ним собственную функцию $(1,0,0, \ldots)$ с собственным значением 1 , получаем полную систему собственных функций оператора $V_{U}$. Пусть теперь $T: F(H) \rightarrow L_{2}\left(Q, \mu_{I / 2}\right)$ - изоморфизм гильбертовых пространств такой, что

$$
\begin{gathered}
T:(1,0,0, \ldots) \mapsto 1 \\
T:\left(0, \ldots, z_{1}^{n} \vee \cdots \vee z_{n}^{n}, 0, \ldots\right) \mapsto a_{1}^{*}\left(\operatorname{Re}\left(z_{1}^{1}\right) \times \operatorname{Im}\left(z_{1}^{1}\right)\right) \circ \cdots \circ a_{1}^{*}\left(\operatorname{Re}\left(z_{n}^{n}\right) \times \operatorname{Im}\left(z_{n}^{n}\right)\right)(1) .
\end{gathered}
$$

При этом изоморфизме операторы $a(f), a^{*}(f)$ перейдут в операторы $a_{1}(f), a_{1}^{*}(f)$, а собственные функции оператора $V_{U}$ перейдут в полиномиальные функции, которые являются собственными функциями преобразования Фурье.

ЛЕмма 3. Пусть симплектическое преобразование $U$ унитарно и оператор $V_{U}$ сплетающий для $U$ в пространстве Фока, т.е. $V_{U}$ удовлетворяет соотношению $V_{U} \circ a(z) \circ V_{U}^{-1}=a(U(z))$. Тогда оператор $T \circ V_{U} \circ T^{-1}$ является сплетающим $в$ картине Винера-Сигала-Фока или, что то же самое, определяет соответствующее унитарное преобразование Боголюбова. 
ДокАЗАТЕЛЬСТВо. Пусть $U$ представим матрицей

$$
\left(\begin{array}{cc}
A & -B \\
B & A
\end{array}\right)
$$

Тогда $U$ индуцирует автоморфизм в алгебре операторов (преобразование Боголюбова), который в картине Винера-Сигала-Фока имеет вид $a_{1}(z) \mapsto a_{1}\left(U^{*}(z)\right)$ или, что то же самое в терминах $\widehat{q}_{x}, \widehat{p}_{x}$,

$$
\left(\begin{array}{c}
\widehat{q}_{a} \\
\widehat{p}_{a}
\end{array}\right) \mapsto\left(\begin{array}{c}
\widehat{q}_{A^{*}(a)}+\widehat{p}_{-B^{*}(a)} \\
\widehat{q}_{B^{*}(a)}+\widehat{p}_{A^{*}(a)}
\end{array}\right) .
$$

В силу унитарности $U$ автоморфизм, задаваемьй оператором $U^{-1}$ в алгебре операторов $a(z)$, действуюших в пространстве раторов $a_{1}(z)$ пространства Винера-Сигала-Фока. Лемма доказана.

Из рассмотрения картины Фока, в которой унитарные преобразования Боголюбова, отвечающие унитарным преобразованиям симплектического пространства, имеют простой вид, получаем следующую теорему.

ТЕОрема 3. Пусть последовательность унитарных операторов $\left\{U_{n}\right\}$ сходится в сильной операторной топологии (в топологии поточечной сходимости) к унитарному оператору $U$. Тогда последовательность соответствующих унитарных преобразований Боголюбова $V_{U_{n}}$ сходится $\kappa V_{U}$ в сильной операторной топологии.

ДокАЗАТЕльство. Докажем, что для произвольного элемента пространства Фока $h$ последовательность $\left\{V_{U_{n}}(h)\right\}$ сходится по норме к $\left\{V_{U}(h)\right\}$. В силу унитарности $U_{n}$ и $U$ достаточно доказать это только для элементов $h$ финитных и конечного ранга. А для таких $h$ утверждение очевидно. Теорема доказана.

Последняя теорема позволяет получить явные формулы для унитарных преобразований Боголюбова, отвечаюших унитарньм преобразованиям $H$ в картине Винера-Сигала-Фока. Пусть $H=Q \times P$ и $Q=Q_{1} \times Q_{2}$. Тогда $H=H_{1} \times H_{2}$, где $H_{i}=Q_{i} \times P_{i}$, $i=1,2$, и унитарньй оператор $U$ оставляет инвариантными $H_{1}$ и $H_{2}$. Если рассматривать $H_{i}$ как симплектические пространства, то в качестве преобразования Боголюбова $V_{U}$ можно взять тензорное произведение

$$
V_{U}=V_{U \mid H_{1}} \otimes V_{U \mid H_{2}}, \quad \text { где } V_{U} \mid H_{i}: L_{2}\left(Q_{i}, \mu_{1 / 2}\right) \rightarrow L_{2}\left(Q_{i}, \mu_{1 / 2}\right) .
$$

Так как унитарное преобразование Боголюбова определено с точностью до единичного множителя, можно было взять его таким образом, что $V_{U}(1)=1$. Единица здесь играет роль вакуумного вектора в пространства Фока. Резюмируя, получаем, что

$$
V_{U}(f)=\lim _{n \rightarrow \infty} V_{U_{n}}\left(f\left(P_{n}(\cdot)\right)\right.
$$

где $U_{n}$ и $P_{n}$ определяются ниже. Пусть $\left\{e_{i}\right\}$ - последовательность собственных векторов $U$. Определим семейство $T_{n}$ подпространств пространства $Q$ таких, что

$$
T_{n}=\operatorname{Link}\left\{\operatorname{Re}\left(e_{i}\right), \operatorname{Im}\left(e_{i}\right), i=1, \ldots, n\right\} .
$$


Обозначим через $P_{n}$ ортогональньй проектор на подпространство $T_{n}$. Пусть $U_{n}-$ произвольный унитарньй оператор, удовлетворяющий следующим условиям: $n$ первых собственных векторов оператора $U_{n} \mid T_{n}$ являются собственными векторами оператора $U$ с теми же собственными значениями и $U_{n} \mid\left(T_{n}\right)^{\perp}=\mathrm{id}$. Мы предполагаем, что $f(\cdot)-$ гладкая быстро убывающая функция. Так как всякий унитарньй оператор обладает спектральным представлением (т.е. $\left.U=\int_{-\infty}^{+\infty} \exp i \phi d E_{\phi}\right)$, то $V_{U}$ есть предел унитарных операторов рассмотренного выше типа (обладающих полной системой собственных функций).

4. Некоторые подгруппы симплектической группы. Вопрос о существовании преобразований Боголюбова решает теорема Шейла (см., например, [8]), а именно, для преобразования $S$ существует унитарное преобразование Боголюбова тогда и только тогда, когда $\left(S^{*} S\right)^{1 / 2}-I$ - оператор Гильберта-Шмидта. Обозначим множество всех симплектических операторов $S$ таких, что оператор $\left(S^{*} S\right)^{1 / 2}-I$ ядерный, через $S_{1}$, а множество всех симплектических операторов $S$ таких, что $\left(S^{*} S\right)^{1 / 2}-I$ - оператор Гильберта-Шмидта, - через $S_{2}$.

ТеОрема 4. Пусть $g \in S_{i}$. Тогда существуют унитарные преобразования $U_{1}, U_{2}$ и преобразование $W \in S_{i}$ вида $W=\left(d^{-1}, 0,0, d^{*}\right)$ такие, что выполнено равенство $g=U_{1} \circ W \circ U_{2}$.

ДокАЗАТЕЛЬСтво. Обозначим $U_{1}, U_{2}, W$ соответственно через $U_{1}(g), U_{2}(g), W(g)$. Пусть

$$
J: Q \times P \ni x \times y \mapsto-y \times x \in Q \times P
$$

- умножение на мнимую единицу. Тогда условие симплектичности $g$ имеет вид $J \dot{g} \dot{J}^{-1}=$ $g^{*-1}$. Из симплектичности самосопряженного отображения $g^{*} g$ следует, что если $e-$ собственньй вектор отображения $g^{*} g$ с собственным значением $\lambda$, то $J(e)$ - тоже собственньй вектор с собственньм значением $1 / \lambda$. Отсюда следует, что существуют унитарное отображение $V$, подпространство $H$ пространства $M$ такое, что $H=M \times J M$, оператор $t: M \rightarrow M$ такой, что $V(Q)=M$ и

$$
g^{*} g: Q \times P \ni x \times y \mapsto t \circ V(x) \times J \circ t^{-1} \circ V(y) \in M \times M=H .
$$

Tak как

$$
\left(g^{*} g\right)^{1 / 2}: Q \times P \ni x \times y \mapsto t^{1 / 2} \circ V(x) \times J \circ t^{-1 / 2} \circ V(y) \in M \times M=H,
$$

то $\left(g^{*} g\right)^{1 / 2}$ симплектическое. Пусть $g=O \circ\left(g^{*} g\right)^{1 / 2}$, где $O$ ортогонально. Тогда из группового закона следует, что $O$ унитарньй. Отсюда следует заключение теоремы.

Имеет место и обратное утверждение: если $g$ представим в виде $g=U_{1} \circ W \circ U_{2}$, где $W \in S_{i}$, то $g \in S_{i}$. Это замечание позволяет определить множества $S_{i}$ как множества симплектических преобразований, представимых в виде $U+K$, где оператор $U$ унитарньй, а $K$ - оператор Гильберта-Шмидта или ядерный. Отсюда следует, что $S_{i}$ являются подгруппами симплектической групшы. Это утверждение позволяет написать явную формулу для унитарного преобразования Боголюбова, соответствующего симплектическому преобразованию из $S_{1}$. 
5. Связь с подобными результатами. В книге [2] определяется другое проективное представление симплектической группы конечномерного симплектического пространства, которое оказьвается $\psi$-эквивалентным рассмотренному вьше (о понятии $\psi$-эквивалентности подробнее см. [9]).

ОПРЕДЕЛЕниЕ. Представления $T(\cdot), T_{1}(\cdot)$ групы $G$ называются $\psi$-эквивалентными, если существует автоморфизм $\psi$ групшы $G$ такой, что $T(\psi(\cdot))$ и $T_{1}(\cdot)$ эквивалентны в обычном смысле.

Если в качестве сплетающего оператора взять тождественный, а автоморфизм симплектической группы $\psi((\alpha, \beta, \gamma, \delta))=(\delta, \gamma, \beta, \alpha)$, то эти проективные унитарные представления будут $\psi$-эквивалентны. Под эквивалентностью проективных унитарных представлений подразумевается коммутативность соответствующей диаграммы с точностью до умножения на комплексное число с единичньпм модулем, зависящее от элемента групшы. Вопрос об эквивалентности этих представлений решает

ТЕОРема 5. Рассмотренная пара представлений конечномерной симплектической группы не әквивалентна в обычным смысле.

ДокАЗАТЕЛЬСтво. Операторы эквивалентных представлений, соответствующие одному и тому же элементу группы, имеют одинаковый спектр. Рассмотрим симплектическое преобразование

$$
\left(\begin{array}{cc}
\cos (\varphi) & -\sin (\varphi) \\
\sin (\varphi) & \cos (\varphi)
\end{array}\right),
$$

т.е. так называемьй “поворот". Спектр соответствующего унитарного преобразования Боголюбова имеет вид $\{\exp i n \varphi, n=0,1, \ldots\}$. Так как на унитарных преобразованиях автоморфизм $\psi$ реализуется оператором взятия обратного, то у оператора второго представления спектр $\{\exp -i n \varphi, n=0,1, \ldots\}$. Так как при подходящем $\varphi$ спектр различен, то теорема доказана.

6. Коцикл проективного представления и подъем проективного представления до обычного. Максимальное изотропное подпространство $H$, т.е. максимальное подпространство $K$ такое, что для всех $x, y \in K$ вьполнено равенство $B(x, y)$ $=0$, назьвают лагранжсевой плоскостью (подробнее см. [2]). Удобно представлять лагранжеву плоскость как замыкание вещественной линейной оболочки комплексного ортогонального базиса. В случае конечномерного пространства $H$ индекс Маслова трех лагранжевых плоскостей $l_{1}, l_{2}, l_{3}$ определяется как сигнатура квадратичной формы $B(x, y)+B(y, z)+B(z, x)$ на пространстве $l_{1} \times l_{2} \times l_{3}$ и обозначается через $\tau\left(l_{1}, l_{2}, l_{3}\right)$. Основное свойство индекса Маслова, так называемое иепное правило, имеет вид

$$
\tau\left(l_{1}, l_{2}, l_{3}\right)=\tau\left(l_{1}, l_{2}, l_{4}\right)+\tau\left(l_{2}, l_{3}, l_{4}\right)+\tau\left(l_{3}, l_{1}, l_{4}\right) .
$$

Доказательство см. в книге [2].

Если есть унитарное проективноепредставление $T(\cdot)$ групшы $G$, то для каждого элемента $g \in G$ можно взять определенньй оператор $T(g)$; тогда комплекснозначная функция $c(\cdot, \cdot)$, равная по модулю единице, такая, что вьполняется равенство

$$
T\left(g_{1}\right) \circ T\left(g_{2}\right)=c\left(g_{1}, g_{2}\right) T\left(g_{1} g_{2}\right)
$$


назьвается коииклом проективного унитарного представления. Коцикл удовлетворяет уравнению коцикла

$$
c\left(g_{1}, g_{2}\right) c\left(g_{1} g_{2}, g_{3}\right)=c\left(g_{1}, g_{2} g_{3}\right) c\left(g_{2}, g_{3}\right)
$$

которое следует из ассоциативности умножения. Выберем теперь $V_{g}, g \in S_{1}$, так, чтобы $V_{g}(1)(0)>0$. Так как функция $V_{g}(1)$ представляет собой экспоненту от квадратичной формы, которая продолжается на $Q_{-}$, мы можем естественным образом определить ее значение в точке 0 .

ЛЕмма 4. Пусть $g_{1}, g_{2} \in S_{1}$. Тогда

$$
\begin{aligned}
c\left(g_{1}, g_{2}\right)=c & \left(U_{1}\left(g_{1}\right) \cdot U_{1}\left(W\left(g_{1}\right) \dot{U}_{2}\left(g_{1}\right) g_{2}\right), W\left(W\left(g_{1}\right) \cdot U_{2}\left(g_{1}\right) g_{2}\right)\right) \\
& \times c^{-1}\left(U_{1}\left(W\left(g_{1}\right) \cdot U_{2}\left(g_{1}\right) \cdot g_{2}\right), W\left(W\left(g_{1}\right) \cdot U_{2}\left(g_{1}\right) \cdot g_{2}\right)\right) \\
& \times c\left(U_{2}\left(g_{1}\right) \dot{U}_{1}\left(g_{2}\right), W\left(g_{2}\right)\right) \cdot c^{-1}\left(U_{1}\left(g_{1}\right), W\left(g_{1}\right)\right) \cdot c^{-1}\left(U_{1}\left(g_{2}\right), W\left(g_{2}\right)\right)
\end{aligned}
$$

әде $U_{1}, W, U_{2}$ определены в теореме 3.

ДокАЗАТЕЛЬСТВо следует из уравнения коцикла и из того, что

$$
c\left(W\left(h_{1}\right) \cdot h_{2}, h_{3}\right)=c\left(h_{2}, h_{3}\right), c\left(h_{1}, h_{2} \cdot U\right)=c\left(h_{1}, h_{2}\right)
$$

для симплектических $h_{1}, h_{2}, h_{3}$ и унитарного $U$.

Последняя лемма позволяет выразить значение коцикла в произвольных элементах $S_{1}$ через значения в элементах более простого вида. Справедлива следующая

Лемма 5. Пусть $\operatorname{Re}(a)>0$. Тогдa

$$
\int_{-\infty}^{+\infty} \exp \frac{-a x^{2}}{2} d x=\frac{\sqrt{2 \pi}}{\sqrt{a}}
$$

əде $\arg \sqrt{a} \in[-\pi / 2, \pi / 2]$.

ДокАЗАТЕЛЬСтво следует из теоремы Коши.

ТЕОрема 6. Пусть унитарное преобразование $U=(A,-B, B, A)$, а симплектическое преобразование $W=\left(d^{-1}, 0,0, d^{*}\right)$. Тогда кочикл

$$
c(U, W)=\frac{\left(\operatorname{det}\left(I+\left(d^{*} \cdot d-I\right) \cdot\left(B^{*} B+i A^{*} \cdot B\right)\right)\right)^{1 / 2}}{\left|\operatorname{det}\left(I+\left(d^{*} \cdot d-I\right) \cdot\left(B^{*} B+i A^{*} \cdot B\right)\right)\right|^{1 / 2}}
$$


ДокАЗАТЕЛЬСтво. Будем для простоты считать, что $B$ обратим. В силу выбора унитарных преобразований Боголюбова

$$
c(U, W)=\frac{\left|V_{U \circ W}(1)(0)\right|}{V_{U \circ W}(1)(0)} .
$$

Иначе говоря,

$$
\frac{1}{V_{U \circ W}(1)(0)}=\lim _{n \rightarrow \infty} \frac{\int_{T_{n}} \exp \left(i\left\{\frac{\left\langle\beta_{n}^{-1} \alpha_{n} y, y\right\rangle}{2}\right\}-\frac{\langle y, y\rangle}{2}\right) d y}{\int_{T_{n}} \exp \left(i\left\{\frac{\left\langle\beta_{n}^{-1} \alpha_{n} y, y\right\rangle}{2}\right\}-\frac{\left\langle P_{n} \dot{d} y, P_{n} \cdot d y\right\rangle}{2}\right) d y}
$$

где $P_{n}$ - ортогональньй проектор на $T_{n}=\operatorname{Link}\left\{\operatorname{Re}\left(e_{i}\right), \operatorname{Im}\left(e_{i}\right), i=1, \ldots, n\right\},\left\{e_{i}\right\}-$ собственные вектора $U$, а $\alpha_{n}, \beta_{n}$ - элементы матрицы унитарного оператора, совпадающего на $T_{n}$ с оператором $U$ и тождественного на ортогональном дополнении к $T_{n}$. Учитывая, что

$$
\left(i \beta^{-1} \cdot \alpha-I\right)^{-1}=-\beta^{*} \cdot \beta-i \alpha^{*} \circ \beta
$$

и применяя последнюю лемму, получаем заключение теоремы.

ЗАмЕчАниЕ. В [2] для случая конечномерной симплектической группы и некоторой ветви проективного представления справедливо представление для коцикла

$$
c\left(g_{1}, g_{2}\right)=\exp \frac{i \cdot \pi \tau\left(P, g_{1}(P), g_{1} \cdot g_{2}(P)\right.}{4}
$$

где проективное представление, рассматриваемое в [2], $\phi$-эквивалентно рассматриваемому в работе.

Аналогично, используя явные формулы для операторов представления, можно получить другое представление для коцикла. Если симплектические преобразования $g_{1}=$ $\left(\alpha_{1}, \beta_{1}, \gamma_{1}, \delta_{1}\right), g_{2}=\left(\alpha_{2}, \beta_{2}, \gamma_{2}, \delta_{2}\right)$ околоединичные, то

$$
c\left(g_{1}, g_{2}\right)^{2}=\frac{\left(\operatorname{det}\left(\alpha_{1}+i \cdot \beta_{1}\right) /\left|\operatorname{det}\left(\alpha_{1}+i \cdot \beta_{1}\right)\right|\right) \cdot\left(\operatorname{det}\left(\alpha_{2}+i \cdot \beta_{2}\right) /\left|\operatorname{det}\left(\alpha_{2}+i \cdot \beta_{2}\right)\right|\right)}{\operatorname{det}\left(\alpha_{3}+i \cdot \beta_{3}\right) /\left|\operatorname{det}\left(\alpha_{3}+i \cdot \beta_{3}\right)\right|}
$$

где $g_{1} \cdot g_{2}=\left(\alpha_{3}, \beta_{3}, \gamma_{3}, \delta_{3}\right)$. Справедливость данного представления для коцикла устанавливается путем приближения ядерного оператора конечномерным.

Если дана группа $G$ и ее проективное унитарное представление с некоторой ветвью $T: G \rightarrow U(H)$, коцикл $c: G \times G \rightarrow S^{1}$, то можно построить расширение $\widehat{G}$ такое, что проективное представление $T$ групшы $G$ поднимется до обычного представления $\widehat{T}$ группы $\widehat{G}$. Как множество група $\widehat{G}$ отождествляется с $G \times S^{1}$, а умножение определяется так:

$$
(g, t)\left(g_{1}, t_{1}\right)=\left(g g_{1}, \frac{t t_{1}}{c\left(g, g_{1}\right)}\right)
$$


Эта группа называется расширением Макки групшы $G$. Если положить $\widehat{T}((g, t))=$ $t T(g)$, то получится настоящее (не проективное) представление группы $G$. Если в качестве $G$ взять $S_{1}$, то получится некоторое накрытие, на котором проективное представление будет обычным. В случае $\operatorname{dim} H<\infty$ в [2] строится двулистное накрытие симплектической группы, на которое проективное представление поднимается до обычного. Полученная группа назьвается метаплектической. Для построения метаплектической групшы находят функцию $f: G \rightarrow S^{1}$ такую, что верно равенство

$$
c\left(g_{1}, g_{2}\right)^{2}=\frac{f\left(g_{1}\right) f\left(g_{2}\right)}{f\left(g_{1} g_{2}\right)} .
$$

Лемма 6. Пусть подмнохество в $G \times S^{1}$ вида $\left(g, t_{i}\right), i=1,2$ (m.е. имеет двузначную проекиию на $G),-$ подгруппа. Тогда $t_{1}^{2}=t_{2}^{2}$.

ДокАЗАТЕЛЬСТвО. Пусть для $g, h, p \in G$ выполнено $g \cdot h=p$. Тогдаиз двулистности проекции получаем

$$
\begin{array}{ll}
\left(g, a_{1}\right) \cdot\left(h, b_{1}\right)=\left(p, c_{1}\right), & \left(g, a_{1}\right) \cdot\left(h, b_{2}\right)=\left(p, c_{2}\right) \\
\left(g, a_{2}\right) \cdot\left(h, b_{1}\right)=\left(p, c_{2}\right), & \left(g, a_{2}\right) \cdot\left(h, b_{2}\right)=\left(p, c_{1}\right)
\end{array}
$$

Приравнивая вторые компоненты, вьводим

$$
\frac{a_{1} b_{1}}{c(g, h)}=c_{1}, \quad \frac{a_{1} b_{2}}{c(g, h)}=c_{2}, \quad \frac{a_{2} b_{1}}{c(g, h)}=c_{2}, \quad \frac{a_{2} b_{2}}{c(g, h)}=c_{1} .
$$

Деля соответствующие равенства друг на друга, а затем приравнивая, получаем

$$
\frac{a_{1}}{a_{2}}=\frac{a_{2}}{a_{1}}, \quad \frac{b_{1}}{b_{2}}=\frac{b_{2}}{b_{1}}, \quad \frac{c_{1}}{c_{2}}=\frac{c_{2}}{c_{1}} .
$$

Отсюда получается утверждение леммы.

Эту лемму можно перенести на случай $n$-листного накрытия. Из леммы получаем, что если в расширении Макки есть подгруппа с двузначной проекцией, то существует функция $G: f \rightarrow S^{1}$, для которой при всех $g_{1}, g_{2} \in G_{1}$

$$
c\left(g_{1}, g_{2}\right)^{2}=\frac{f\left(g_{1}\right) f\left(g_{2}\right)}{f\left(g_{1} g_{2}\right)} .
$$

Таким образом, мы доказали следующую теорему.

ТЕОрема 7. Для $S_{1}$ существует подгруппа в расширении Макки, обладающая двулистной проекчией, такая, что проективное представление поднимается на эту подгруппу до обычного.

Но двулистное накрытие существует и для больших подгрупп симплектической группы. 
Теорема 8. Для подаруппы симплектической группь, состоящей из преобразований $g$ таких, что $g=e^{i \cdot \phi}+\varepsilon=(\alpha, \beta, \gamma, \delta)$, әде оператор $\varepsilon$ ядерный, справедливо равенство

$$
c\left(g_{1}, g_{2}\right)^{2}=\frac{f\left(g_{1}\right) f\left(g_{2}\right)}{f\left(g_{1} g_{2}\right)},
$$

əде

$$
f(g)=\frac{\operatorname{det}(\alpha \cos \phi-\beta \sin \phi+i(\alpha \sin \phi+\beta \cos \phi))}{|\operatorname{det}(\alpha \cos \phi-\beta \sin \phi+i(\alpha \sin \phi+\beta \cos \phi))|} .
$$

ДоКАЗАТЕЛЬСТво. Пусть сначала операторы

$$
g_{1}=e^{i \cdot \phi_{1}} \cdot\left(I+\varepsilon_{1}\right), \quad g_{2}=e^{i \cdot \phi_{2}} \cdot\left(I+\varepsilon_{2}\right)
$$

таковы, что $\varepsilon_{1}, \varepsilon_{2}$ конечномерные. Обозначим через $P_{1}$ подпространство $P$ такое, что подпространство $P_{1} \times P_{1}$, обозначаемое как $H_{1}$, содержит образы операторов $\varepsilon_{1}, \varepsilon_{2}$. Так как

$$
c\left(g_{1}, g_{2}\right)=c\left(\left.g_{1}\right|_{H_{1}},\left.g_{2}\right|_{H_{1}}\right) \cdot c\left(\left.g_{1}\right|_{\left(H_{1}\right)^{\perp}},\left.g_{2}\right|_{\left(H_{1}\right)^{\perp}}\right)
$$

и на подгруппе унитарных операторов коцикл равен единице, то

$$
c\left(g_{1}, g_{2}\right)=c\left(\left.g_{1}\right|_{H_{1}},\left.g_{2}\right|_{H_{1}}\right) .
$$

Положим

$$
h(g)=\frac{\operatorname{det}(\alpha+i \cdot \beta)}{|\operatorname{det}(\alpha+i \cdot \beta)|},
$$

тогда

$$
c\left(g_{1}, g_{2}\right)=\frac{h\left(\left.g_{1}\right|_{H_{1}}\right) \cdot h\left(\left.g_{2}\right|_{H_{1}}\right)}{h\left(\left(g_{1} \cdot g_{2}\right) \mid H_{1}\right)} .
$$

Сокращая на операторы вида $e^{i \cdot t}$, получаем нужное утверждение. Если на рассматриваемой подгрупше ввести расстояние

$$
\rho\left(g_{1}, g_{2}\right)=\left|\phi_{1}-\phi_{2}\right|+\left|\varepsilon_{1}-\varepsilon_{2}\right|_{1},
$$

где $|\cdot|_{1}$ - обычная ядерная норма, то представление $f(\cdot)$ окажется непрерывным (предполагается, что в пространстве операторов представления имеется сильная операторная топология). Таким образом, формула распространяется на операторы $g$ с ядерным $\varepsilon$. Теорема доказана.

ЗАмЕчАниЕ. Предыдущая теорема переносится на подгрупу симплектических преобразований, представимых в виде $g=e^{i \cdot t A}+\varepsilon$, где однопараметрическая подгрупша $e^{i \cdot t A}$ имеет тривиальное пересечение с подгрупой $G_{1}$ (состоящей из преобразований, являюшихся суммой тождественного и ядерного), а оператор $\varepsilon$ ядерньй.

Теорема 9. Для группь $S_{1}$ не существует подгруппы расширения Макки, имеющей двулистную проекцию, на которую проективное представление поднимается до обычного. 
ДокАЗАТЕЛЬСтво. Так как у группы унитарных операторов бесконечномерного гильбертова пространства нет нетривиальных характеров и сужение нашего проективного представления на эту подгруппу допускает выделение однозначной ветви, то сужение функции $f$ на группу унитарных операторов тождественно равно 1 . По аналогичным причинам $f(W)= \pm 1$ для $W \in G_{1}, W=\left(d^{-1}, 0,0, d^{*}\right)$. Из того, что операторы представления, соответствующие унитарным преобразованиям, сохраняют вакуумный вектор, а операторы, соответствующие растяжениям сохраняют "значение вакуумного вектора в нуле", следует, что для $g=U \cdot W \cdot V$ вьполнено $f(g)=c(U, W)$. Последняя формула для $f(g)$ не согласуется с тем, что $f(W \cdot g)=f(g)$, поскольку последнее равенство не верно, если взять $W_{2} \cdot U_{1} \cdot W_{1}=U \cdot W \cdot V$, где

$$
\begin{gathered}
W_{2}=\left(\begin{array}{ll}
4 & 0 \\
0 & \frac{1}{4}
\end{array}\right), \quad U_{1}=\left(\begin{array}{cc}
\frac{1}{2} & -\frac{\sqrt{3}}{2} \\
\frac{\sqrt{3}}{2} & \frac{1}{2}
\end{array}\right), \quad W_{1}=\left(\begin{array}{ll}
2 & 0 \\
0 & \frac{1}{2}
\end{array}\right), \\
U=\left(\begin{array}{cc}
-0.0854 & -0.9963 \\
0.9963 & -0.0854
\end{array}\right), \quad W=\left(\begin{array}{cc}
0.2286 & 0 \\
0 & 4.3748
\end{array}\right), \quad V=\left(\begin{array}{cc}
0.3932 & 0.9194 \\
-0.9194 & 0.3932
\end{array}\right) .
\end{gathered}
$$

Предполагается, что приведенные вьше симплектические преобразования задаются указанными матрицами на общем конечномерном подпространстве симплектического пространства, а на ортогональном дополнении к нему они тождественны. Теорема доказана.

Автор выражает благодарность О.Г. Смолянову за постановку задачи и ее обсуждение.

\section{СПИСОК ЦИТИРОВАННОЙ ЛИТЕРАТУРЫ}

[1] Купш И., Смолянов О.Г. Реализация в пространствах типа Винера-Сигала-Фока унитарных преобразований, порождающих преобразования Боголюбова // Докл. РАН. 2000. T. 371. № 1. C. 21-25.

[2] Лион ЖК., Вернь М. Представление Вейля, индекс Маслова и тэта-ряды. М.: Мир, 1983.

[3] Ленг С. $S L_{2}(R)$. М.: Мир, 1977.

[4] Березин Ф. А. Метод вторичного квантования. М.: Наука, 1965.

[5] Далецкий Ю. Л., Фомин С. В. Меры и дифференциальные уравнения в бесконечномерных пространствах. М.: Наука, 1983.

[6] Тверитинов И. Д. Преобразования Боголюбова, соответствующие симплектическим преобразованиям // Вестн. МГУ. Сер. 1. Матем., мех. 2002. № 4. С. 9-14.

[7] Ottesen J.T. Infinite-Dimensional Groups and Algebras in Quantum Physics. Berlin: Springer 1995 .

[8] Baez J. C., Segal I. E., Zhou Z. Introduction to Algebraic and Constructive Quantum Field Theory. Princeton: Princeton Univ. Press, 1992.

[9] Березин Ф. А. Несколько замечаний о представлении соотношений коммутации // УМН. 1969. Т. 24. № 4. C. $65-88$. 